\title{
LA ORGANIZACIÓN IBEROAMERICANA DE SEGURIDAD SOCIAL
}

\author{
CARLOS JAVIER SANTOS GARCÍA
}

Director Ejecutivo de la OISS

\section{RESUMEN}

El objetivo principal de la OISS es la promoción del bienestar económico y social de los países Iberoamericanos, mediante la coordinación, intercambio y aprovechamiento de sus experiencias mutuas en Seguridad Social.

Para ello, el organismo dispone de una programación sistemática en los ámbitos de formación, asistencia técnica, visitas interinstitucionales para el intercambio de experiencias, reuniones y conferencias y publicaciones. También actúa como asesor especializado en el área de protección social para MERCOSUR y la Comunidad Andina y se hace cargo de mandatos específicos de las Cumbres de Jefes de Estado y de Gobierno apoyando la función de coordinación e impulso que asume la SEGIB.

En este terreno merece una especial mención el impulso y coordinación prestados para la elaboración del Convenio Iberoamericano de Seguridad Social, ratificado por la XVII Cumbre Iberoamericana de Jefes de Estado y de Gobierno, que proyecta su acción directa sobre la defensa de los derechos sociales de los trabajadores migrantes en los países de Iberoamérica.

Entre las últimas acciones realizadas, destaca también la promoción del la celebración del II Congreso de Prevención de Riesgos en Iberoamérica, Prevencia 2007, en cuyo marco se han requerido a la O.I.S.S. para abordar dos importantes proyectos:

Elaboración de una Estrategia Iberoamericana en materia de Seguridad y Salud

Creación y mantenimiento de un Observatorio de Siniestralidad en la Region.

\section{PALABRAS CLAVES}

Seguridad Social, Prevención de Riesgos laborales, Seguridad y salud en el trabajo, Iberoamérica, América Latina.

\begin{abstract}
The main objective of the OISS (Ibero-American Organization of Social Security) is promoting the economic and social welfare of the Ibero-American countries, through the coordination, exchange and utilization of their mutual experiences about Social Security.
\end{abstract}

To that end, the Organization has a systematic programming in the areas of training, technical assistance, visits to exchange experiences, meetings, conferences and publications. It also acts as a specialized advisor in the area of social protection for MERCOSUR and the Andean Community and it asumes some responsabilities for specific tasks of the Summit of Heads of State and government to support the coordinating role and motivation provided by the SEGIB.

In this sense, it deserves a special mention the coordination and support provided for the development of the Ibero-American Agreement of Social Security, ratified by the XVII Ibero-American Summit of Heads of State and Government, which is planning its direct action on the defense of the social rights of migrant workers in the countries of IberoAmerica.

Among the latest actions implemented, it is worth mentioning the celebration of the Second Congress about Occupational Risk Prevention in Latin America, Prevencia 2007, where the OISS has been required to tackle two important projects:

Developing an Iberoamerican Strategy about Security and Healthy

Creation and maintaining an Occupational Risk Observatory in the Region.

\section{KEY WORDS}

Social Security, Occupational risks preventio, Security and healthy, Ibero America. 


\section{¿QUÉ ES LA OISS?}

Según la definen sus Órganos Estatutarios, la Organización Iberoamericana de Seguridad Social (OISS) es un organismo internacional, de carácter técnico y especializado, que tiene como finalidad promover el bienestar económico y social de los países iberoamericanos y de todos aquellos que se vinculan por el idioma español y portugués mediante la coordinación, intercambio y aprovechamiento de sus experiencias mutuas en Seguridad Social.

Sus primeros antecedentes datan del I Congreso Iberoamericano de Seguridad Social, en cuyo seno se creó una Secretaría de apoyo a ulteriores congresos que recibiría el nombre de Comisión Iberoamericana de Seguridad Social. Pero fue ya en el transcurso del II Congreso celebrado en Lima (Perú) en 1954 donde, con la presencia de la mayoría de Estados de la Región junto a representantes de la OIT y la AISS, quedó aprobada la Carta Constitutiva de la OISS.

\section{FUNCIONES}

La misión o función principal de la OISS es promover el desarrollo progresivo de la protección social y la máxima ampliación de su campo de acción sobre los ciudadanos iberoamericanos. Se trata, por tanto, de un órgano permanente de información y coordinación de experiencias, que promueve el estudio y la investigación de los sistemas de Seguridad Social, y que capacita al personal de las Instituciones miembros y les presta la asistencia técnica que precisen.

Igualmente, debe proponer los medios adecuados para que sus miembros se presten asistencia técnico-social recíproca y ejecuten planes de acción común. En este sentido, actúa como facilitador de la ejecución de programas de cooperación y desarrollo que otras Instituciones pretendan llevar a cabo en su ámbito de acción, siempre en el área de protección social.

Otra labor importante que cumple la OISS es el mantenimiento de relaciones con otros organismos internacionales relacionados con la Seguridad Social, suscribiendo acuerdos y convenios de cooperación, y promoviendo la adopción de normas internacionales que faciliten la coordinación y favorezcan la internacionalización del Derecho de la Seguridad Social.

\section{ORGANIGRAMA}

\section{Estructura de la OISS}

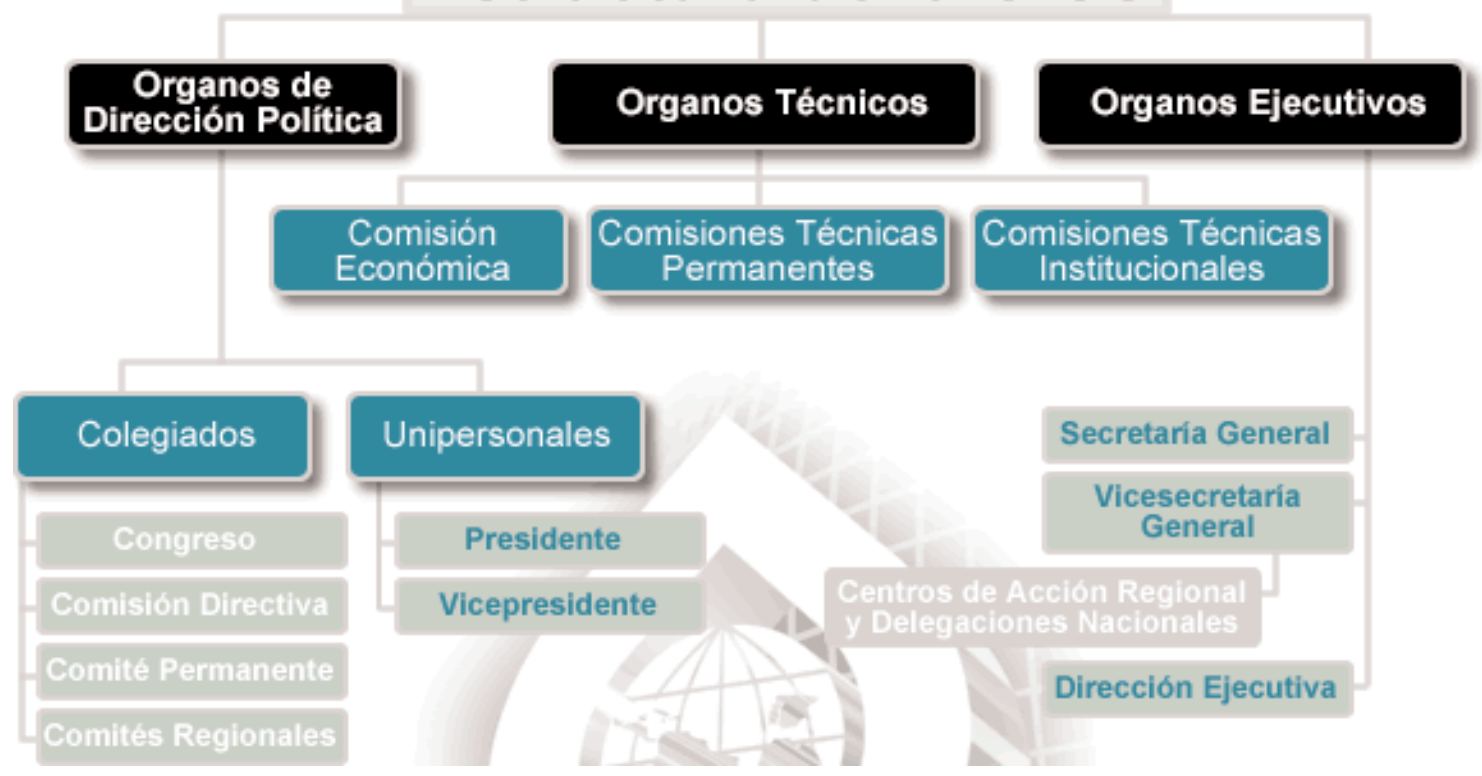




\section{SOCIOS DE LA OISS}

Como Organización de base asociativa, la OISS está constituida actualmente por más de 180 Instituciones entre miembros titulares y asociados, incluyendo 22 gobiernos de Estados Iberoamericanos. Los socios titulares son los gobiernos de los Estados y las instituciones gestoras de regímenes obligatorios de seguros sociales, previsión social y seguridad social.

En cambio, los asociados son instituciones que representan sectores profesionales, laborales, docentes o de investigación, que persigan fines relacionados con la Seguridad Social. Estos miembros no tienen derecho a voto ni participan en los órganos de gobierno.

\section{¿QUÉ SERVICIOS OFRECE LA OISS?}

\section{Programas especiales:}

\section{Convenio Iberoamericano de Seguridad Social}

La XV Cumbre Iberoamericana de Jefes de Estado y de Gobierno, reunida en Salamanca en 2005, acogió la iniciativa de la sesión preparatoria que pocos días antes habían celebrado los Ministros y Máximos responsables de Seguridad Social en su $\mathrm{V}$ Conferencia sectorial, en orden a iniciar el proceso de negociación de un Convenio Iberoamericano de Seguridad Social, concebido como un instrumento jurídico de la Comunidad Iberoamericana para garantizar los derechos sociales de los trabajadores migrantes en la Región.

La Cumbre encomendó a la OISS y a la Secretaría General Iberoamericana $(\boldsymbol{S E G I B})$, el apoyo, preparación y suscripción de este importante instrumento internacional, convirtiéndose desde entonces en uno de los objetivos principales de la Organización, dado el importante número de beneficiarios a los que se dirige, entre trabajadores migrantes y familiares. Se trata, sin duda, de un hito fundamental de cara a consolidar la cohesión social en la Comunidad Iberoamericana, en cuyo ámbito posiblemente sea el primer instrumento jurídico directamente exigible por los ciudadanos.

En este sentido, la OISS ha puesto al servicio de esta idea toda la experiencia obtenida tanto en la Unión Europea, como a través de la labor realizada respecto al Acuerdo Multilateral de Seguridad Social del MERCOSUR y el Instrumento Andino de Seguridad Social, como antecedentes más inmediatos de este nuevo Convenio.

Por eso, ha prestado el apoyo técnico en torno a la elaboración y negociación del Convenio, a través de un proceso ampliamente participativo en el que se han celebrado multitud de Reuniones Técnicas (Montevideo, Santiago de Chile y Sao Paulo), todas anteriores a la VI Conferencia de Ministros y Máximos Responsables del pasado mes de julio, donde se acordó el texto a elevar a la Cumbre de Jefes de Estado y de Gobierno.

\section{Conferencia Iberoamericana de Minist Responsables de Seguridad Social}

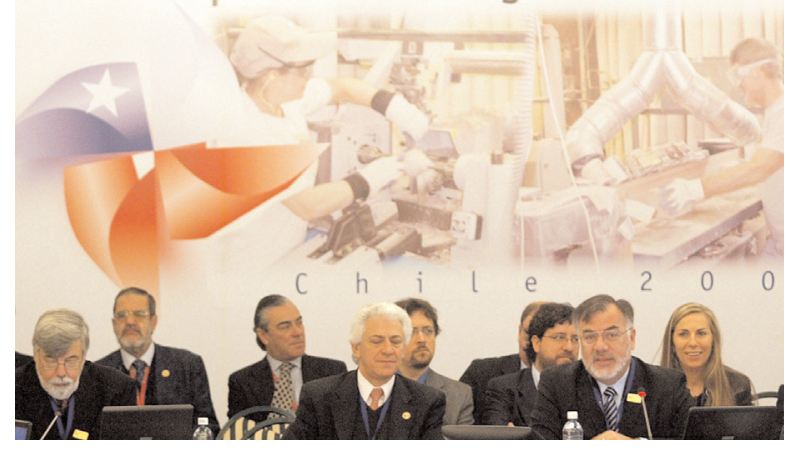

El texto del Convenio está basado en los principios de Igualdad de trato a los nacionales de los países de la Comunidad Iberoamericana, inclusión de trabajadores dependientes y no dependientes, extensión del Convenio a las prestaciones económicas por vejez, invalidez y supervivencia, vigencia de disposiciones más favorables de Convenios anteriores, garantía de derechos adquiridos y en curso de adquisición, y colaboración administrativa y técnica entre Instituciones.

Recientemente, el Convenio ha sido elevado a la XVII Cumbre Iberoamericana de Jefes de Estado y de Gobierno celebrada en Santiago de Chile, y en ella ha sido ratificado, señalando el acuerdo de la Cumbre que los países miembros adoptan el texto del Convenio Multilateral Iberoamericano de Seguridad Social y se comprometen a impulsar los procedimientos internos para su pronta entrada en vigencia.

2. Impulso al desarrollo de las Políticas de Seguridad y Salud

Un paso importante para el desarrollo de políti- 
cas en la materia han sido los acuerdos y documentos surgidos del Congreso de Prevención de Riesgos laborales en Iberoamérica, Prevencia, del cual ya se han celebrado dos ediciones, Buenos Aires en 2006 y Cádiz en 2007.

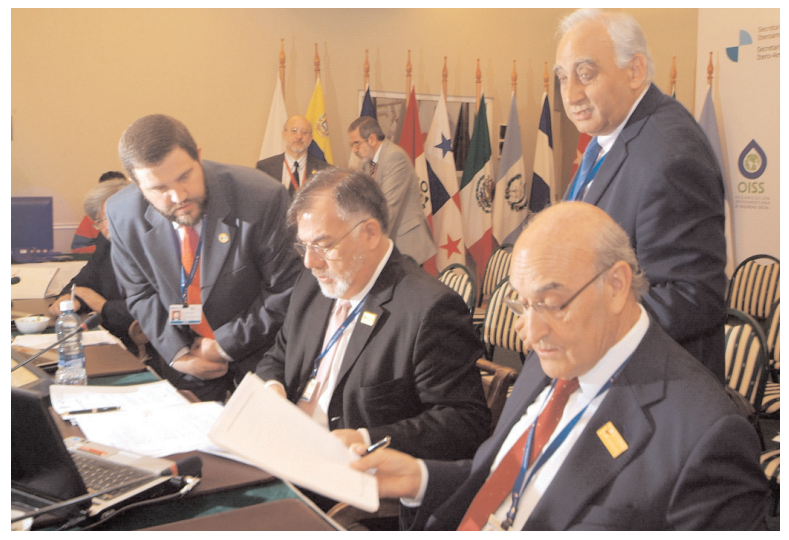

Firma de Declaración de Iquique. VI Conferencia Iberoamericana de Ministros Responsables de Seguridad Social.

De la primera edición surgió la Declaración Iberoamericana sobre Seguridad y Salud en el Trabajo, en la que se apoyó la creación del Observatorio regional sobre prevención de riesgos laborales, se ofrecieron propuestas específicas de actuación dirigidas a los sectores y colectivos más desfavorecidos ante la siniestralidad laboral, y se insistió en la formación y en la educación para crear una verdadera cultura preventiva en la Región.

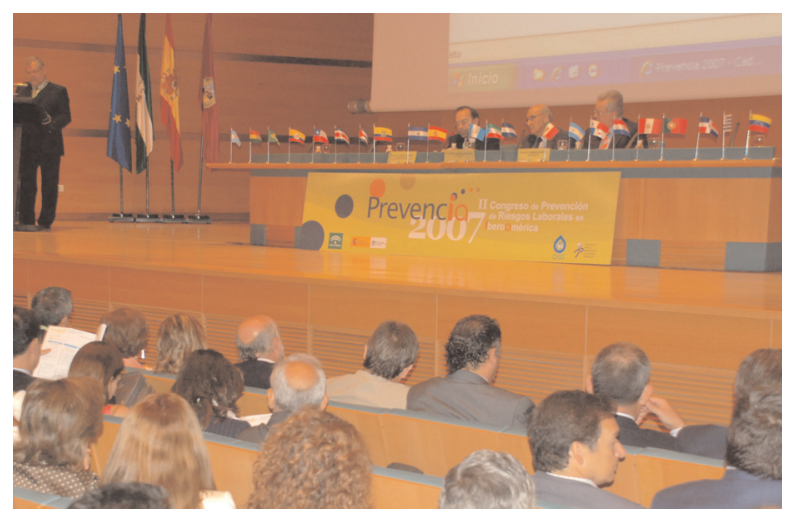

Sesión Inaugural del Congreso.

Por su parte, en Prevencia 2007 se aprobó la Declaración de Cádiz, en la que los asistentes avalaron nuevamente la iniciativa de la OISS para crear un Observatorio de Siniestralidad Laboral en Iberoamérica, a fin de disponer de un sustrato informativo riguroso que permita conducir y priorizar las políticas públicas de la manera más coherente a la realidad de cada país y de la propia Región en conjunto. Además, la Declaración encomendó a la propia OISS el impulso de sucesivas ediciones de Prevencia y la incorporación de programas de formación y asistencia técnica a su planificación bienal de actividades.

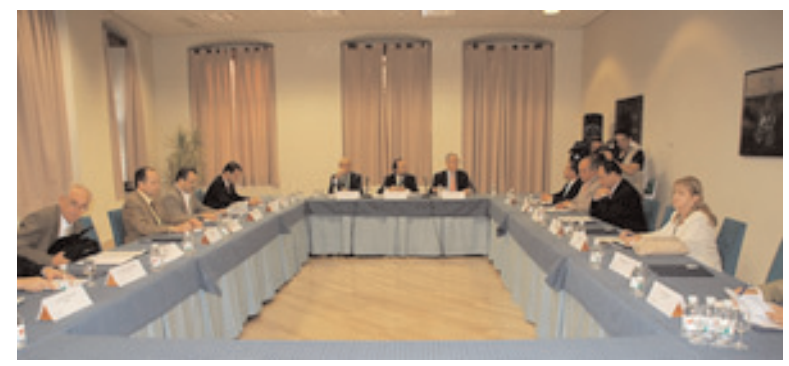

Reunión de Ministros, Viceministros y Máximos Responsables Iberoamericanos de Seguridad y Salud en el Trabajo durante la realización del Congreso

De la Reunión de Ministros celebrada en Cádiz surgió un Acuerdo de Voluntades en el que se expresó el apoyo a los objetivos del Congreso, animando a sus promotores a continuar con la difusión de la cultura preventiva, a fomentar el diálogo social y a gestionar el conocimiento preventivo a favor del trabajo decente.

Además, encomendó a la OISS el desarrollo de una Estrategia Iberoamericana de Seguridad y Salud en el Trabajo que ofrezca respuestas concretas a los actuales problemas específicos de la Región, y que una vez consensuada sea sometida a la Conferencia de Ministros de la materia y, en su caso, elevada a una próxima Cumbre Iberoamericana de Jefes de Estado y de Gobierno.

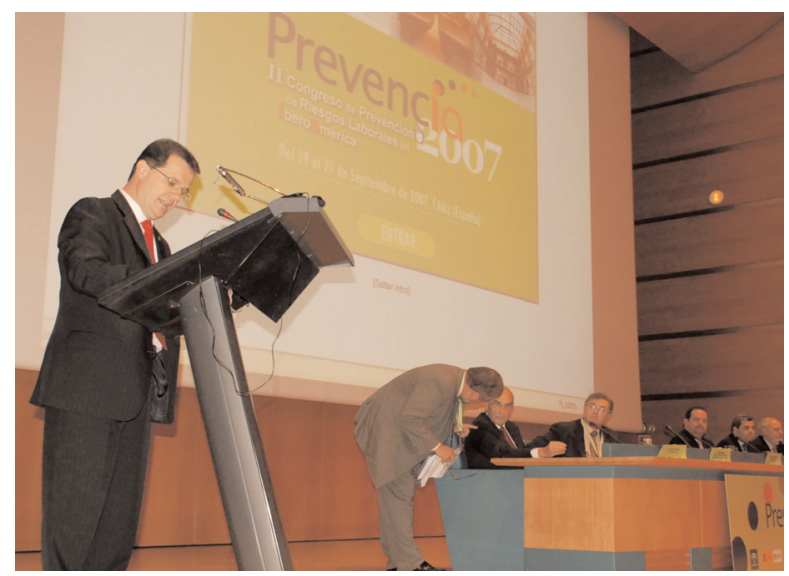

El Viceministro de Trabajo de Brasil y Presidente de la OISS, D. Carlos Gabas al inicio de la Lectura del Acuerdo de Voluntades. 
Otro frente de trabajo lo constituye la implementación de una estructura estable de formación, información y servicios a través del portal www.serviprevencia.org, que tiene el objetivo de establecer una red permanente y gratuita de servicios compartidos en materia de Prevención de Riesgos Laborales entre Instituciones Iberoamericanas. En este portal pueden encontrarse multitud de documentos, noticias, legislación, eventos, estadísticas, bases de datos de expertos en las distintas especialidades, soportes formativos y divulgativos, metodologías de valoración de riesgos laborales y protocolos de actuación en la empresa, cursos gratuitos on-line, y servicio de consultas relacionadas con la Prevención.

El tercer pilar a destacar dentro del desarrollo de políticas de seguridad y salud es la formación de especialistas y la difusión de la cultura preventiva, a través del Master en Prevención y Protección de Riesgos Laborales, configurado por la OISS y la Universidad de Alcalá de Henares, pero además también junto a soportes y materiales formativos, cursos on-line, material audiovisual, material didáctico para introducir la prevención en la escuela y materiales de divulgación en múltiples formatos.

Como cuarto aspecto del impulso a las políticas de seguridad y salud, y habiendo sido avalado por el plenario del II Congreso de Prevención de Riesgos Laborales en Iberoamérica, está el proyecto para la Construcción de un Observatorio de Prevención y Protección de Riesgos Laborales en Iberoamérica, que incorpore estadísticas del empleo formal e informal, a fin de disponer de un sustrato informativo riguroso que permita conducir y priorizar las políticas públicas de la manera más coherente a la realidad de cada país y de la propia Región en su conjunto.

\section{Programa Eurosocial}

Surge tras la Cumbre Unión Europea-América Latina de trabajadores, como contribución al establecimiento de condiciones que favorezcan la cohesión social, y para facilitar la transferencia de experiencias y conocimientos técnicos entre Administraciones Públicas de Europa y Latinoamérica. El ámbito temporal en que se desarrolla es de 4 años, y se estructura en torno a áreas temáticas, de justicia, educación, empleo, fiscalidad y salud. La OISS participa como miembro asociado en el Consorcio de Fiscalidad, en lo que se refiere a cotizaciones sociales, y a su vez, es miembro titular del Consorcio de Salud para el desarrollo de la cobertura en salud.

\section{Programa "Personas Mayores, dependencia y servicios sociales en los países del Cono Sur"}

$\mathrm{Su}$ objetivo es generar un espacio de trabajo conjunto que contribuya a mejorar la calidad de vida de la población mayor, desarrollando dispositivos de protección social y de atención de las situaciones de dependencia. Para ello se pretende crear un Observatorio Regional y un Portal de Mayores, apoyar las líneas de acción que cada país defina, y facilitar apoyo a estudios e investigaciones.

La primera fase del proyecto se llevó a cabo por Instituciones argentinas, chilenas, brasileñas, uruguayas y paraguayas durante el año 2006. Del trabajo surgió la redacción de un documento común que recoge propuestas de líneas de trabajo y actuaciones concretas para avanzar en la mejoría de las condiciones de vida del colectivo. La intención es ampliar el proyecto a Bolivia, Colombia, Costa Rica, Cuba, Ecuador y México.

\section{Centro Iberoamericano para la Autonomía Personal y Ayudas Técnicas (CIAPAT)}

Ubicado en Buenos Aires, este centro tiene como objetivos facilitar el conocimiento y la utilización de tecnologías de apoyo, favorecer la innovación en diseños pensados para todos, servir de referencia en accesibilidad a nivel de Iberoamérica, favorecer la participación activa de todos los usuarios, y ofrecer formación, información y asesoramiento.

Se dirige a adultos mayores y personas con discapacidades, a profesionales, investigadores, empresarios, diseñadores, entidades y personas relacionadas con los campos de la arquitectura, el diseño, las ayudas técnicas y el mercado de las tecnologías de la rehabilitación.

Los servicios que contempla son fundamentalmente de información y asesoramiento técnico en accesibilidad, comunicación, movilidad, adaptación de puestos de trabajo... con acceso a bases de datos de productos y biblioteca técnica especializada.

\section{Programa Red Social}

Dirigido prioritariamente a personas ciegas o con enfermedades que impidan el correcto funcionamiento de la visión, tiene como objetivo mejorar su calidad de vida y niveles de participación económica mediante la utilización de nuevas tecnologías de la información y la comunicación. Cuenta con una plataforma interactiva, red de centros, materiales de 
orientación y capacitación, guías de buenas prácticas, o promoción del empleo.

Hasta el momento se ha conseguido la capacitación internacional para formadores de la Red de Centros de Recursos, impartidas a través de ponencias y talleres, la apertura del Centro de Recursos de La Paz, y está en proceso de implementación el Centro de Recursos de Buenos Aires.

\section{Proyecto BUSS: "Base única de la Seguri- dad Social de MERCOSUR"}

Se trata de analizar con mayor profundidad las semejanzas y asimetrías de los sistemas de Seguridad Social, y perfeccionar los métodos de coordinación. Se ha trabajado en el desarrollo del sistema de transferencia y validación de datos de la Seguridad Social, y en obtener un procedimiento ágil y seguro para la tramitación de las Prestaciones.

En este sentido, los avances producidos son la ordenación del proceso de capacitación del personal técnico y administrativo de las distintas Instituciones Gestoras, y la difusión externa del proyecto a autori- dades de las Instituciones Gestoras, los Representantes Gubernamentales, a la población en general y a países ajenos de MERCOSUR valorándose la posibilidad de ofrecer convenios de cooperación internacional.

\section{Ciberamérica}

Desde 2005, la OISS ha tenido una participación muy activa a la hora de aportar materiales a este portal, como el BISSI, incluido en la biblioteca virtual del portal, la administración del canal específico de ciberamerica.org denominado Seguridad Social, la inclusión de enlaces con los diferentes Ministerios de Trabajo y Seguridad Social de los países Iberoamericanos, una amplia oferta formativa sobre la materia, el acceso a la Declaración Iberoamericana sobre Seguridad y Salud en Trabajo, la incorporación de noticias de Seguridad Social, las Convocatorias a premio OISS y Premio PREVENCIA 2007, el Proyecto de Ley de reforma de la Seguridad Social española, y la incorporación de enlaces a otras páginas relevantes en materia preventiva y en Seguridad Social.

\section{LÍNEAS DE TRABAJO ESTABLES}

\section{Formación de Recursos Humanos}

De un lado, potenciando la formación universitaria en materia de Seguridad Social a través de los Master de Dirección y Gestión de los Sistemas de Seguridad Social, Prevención y Protección Riesgos Laborales, Dirección y Gestión de Bienestar Social y Servicios Sociales, Dirección y Gestión de Servicios de Salud, y Dirección y Gestión de Planes y Fondos de Pensiones.

Igualmente, constituyen una línea estable los diplomados y e-learning que se realizan íntegramente a través de un Aula Virtual específica en las materias de Gestión de prestaciones económicas de la Seguridad Social, Recaudación de los recursos de la Seguridad Social y Técnicas actuariales y financieras de la Previsión Social.
De otro lado, los Centros de Acción Regional y delegaciones nacionales mantienen una importante actividad docente adecuada a las demandas de las instituciones miembro en su correspondiente ámbito geográfico de actuación, o cualquier otra carencia detectada por la Organización.

2. Cooperación, asistencia y apoyo técnico a la modernización de la gestión de los Sistemas de Seguridad Social

Se viene prestando fundamentalmente a través de las Asesorías Técnicas y las visitas interinstitucionales. Las primeras se desarrollan a requerimiento de los miembros de la OISS sobre cualquier materia que pueda resultar de su interés: acción protectora, sistema de gestión, etc. En cambio, el objetivo de las segundas es intercambiar experiencias y trabajos de interés mutuo. 


\section{Impulso al desarrollo de la Seguridad} Social en Iberoamérica.

En este contexto se está llevando a cabo proyectos tan importantes como el Banco de Información de los sistemas de Seguridad Social (BISSI), que contiene un sustrato informativo comparado de las principales magnitudes que influyen en la evolución de dichos sistemas. Pretende facilitar el conocimiento, apoyo mutuo y asesoramiento técnico entre los Estados, dotando de mayor eficacia y eficiencia a las acciones de cooperación que se articulen para apoyar el desarrollo de la Seguridad Social, permitiendo un conocimiento del entorno, de las características del sistema y de sus alternativas de reforma.

Otro aspecto importante es el apoyo a los procesos de integración regional, destacando la figura del asesor permanente de Seguridad Social de la OISS para MERCOSUR. Es aquí donde surge el Acuerdo Multilateral de Seguridad Social, que pretende atender los efectos sociales de los movimientos migratorios que se producen como consecuencia de los procesos de integración, a fin de evitar las situaciones de desprotección o superprotección que pueden producirse.

En tercer lugar se encuentra el apoyo en talleres programados prestados en el marco del Pacto Andino, apoyando la elaboración de las propuestas normativas y asesoramiento.

\section{Actividades de estudio, debate e investigación}

La Organización dispone de foros técnicos estables denominados Comisiones Técnicas en los ámbitos de salud, riesgos laborales, servicios Sociales, prestaciones económicas y pensiones y ahorro previsional. Además, también se han creado los grupos de Trabajo Especializados dedicados a temas específicos. Tanto unas como otros pretenden formar, a través del análisis, el debate y el conocimiento comparado, buscando alternativas que contribuyan a fundamentar orientaciones y decisiones.

\section{Relación con otros organismos y acciones de información y divulgación}

Desde la OISS, las relaciones con organismos nacionales e internacionales siempre se han dado desde una participación activa y una colaboración efectiva, apoyando las Conferencias de Ministros de Trabajo y Máximos Responsables de Seguridad Social, o estando presente como observador en las Cumbres de Jefes de Estado y Gobierno que se celebran.

Además, esa misma colaboración se da en cuantos programas de carácter social coordine la SEGIB, y se participa activamente con instituciones miembros y otros Organismos responsables de Protección Social, habiendo suscrito hasta la fecha más de 30 Convenios Internacionales.

Respecto a las actividades de información y divulgación, podemos encontrar entre las publicaciones periódicas el BISSI, la Revista de la OISS, el Boletín informativo de la OISS (BIOISS) y la Agenda Directorio de 2006. Las publicaciones monográficas son: Monografia sobre el diagnóstico de personas mayores en el Cono sur, Compendio de las Ponencias del Seminario Internacional sobre Protección de los Riesgos Laborales, Retos y respuestas hacia una sociedad en cambio, y Conclusiones del I Congreso Iberoamericano de Prevención y Protección de Riesgos Laborales, Prevencia 2006. 